\title{
A Cold-Standby System with Server Failure and Delayed Treatment
}

\author{
R.K. Bhardwaj \\ Department of statistics \\ Punjabi University Patiala-147002
}

\author{
R. Singh \\ Department of statistics \\ Punjabi University Patiala-147002
}

\begin{abstract}
The paper stochastically investigates a two unit cold-standby system with a server subject to failure and getting delayed treatment thereafter. Semi-Markov process is used to develop the system model. The model is analyzed at different regeneration points using regenerative-point technique. The steady-state expressions are derived for various system performance measures such as mean time to failure, availability, busy period of server, expected number of treatments, profit etc. Finally, numerical examples are given to discuss the effect of various parameters on system performance measures.
\end{abstract}

\section{Keywords}

Cold-Standby System, Steady State, Server Failure, Treatment, Semi-Markov Processes.

\section{INTRODUCTION}

The cold-standby redundancy is widely used by researchers to develop probabilistic system models [1-5]. The repairable standby systems have three basic components; the operative units, standby units and the service facility. The instant availability for use of all these constituents ensures the system reliability. In case of cold-standby system, where the risk of failure of standby unit is negligible as compared to that of the operative one, the role of service facility/ server becomes more accountable for system resumption beside its failure. The problem of server failure has been debated by various researchers in the literature [6-11] in connection with queuing models. Relating to system reliability studies the topic remained concealed for a long time. Recently, few researchers [12-13] discussed the problem of server failure for single unit reliability models. The same problem is generalized with standby reliability models in [14-17].

As far as reliability models with server failure are concerned, the researchers who developed standby systems models focused on instant server treatment at its failure. However, in many cases the failed server takes some time to be placed under treatment either due to surveillance failure, communication delays, heavy traffic load or transportation problem etc. The present study investigates a stochastic model for a cold-standby system of two identical units and a service facility, called server. The system starts operating with one unit in operation and other as cold-standby. The standby unit directly switches into operation as and when the operative unit fails and the latter is then taken for repair by the server. If the server fails during work it moves under treatment with some elapsed time. All the switches are instantaneous and perfect. The unit and the server work as new after each repair and treatment respectively. All the random variables describing the model are statistically independent and uncorrelated. The failure time of unit, repair time of unit, waiting time for treatment and treatment time of server follow general probability distribution with different density functions. The study uses semi-Markov theory [18] to develop the system model and investigates the model at different re-generation points using re-generative point technique of renewal theory [19]. It derives expressions for system performance measures such as mean time to failure, availability, busy period, expected number of repairs and treatments and the profit incurred to the system. Finally, a particular case is discussed for illustrating the results and introducing simulation study.

\section{NOTATIONS}

$E / \bar{E}:$ Set of regenerative states/non regenerative states.

$O$ : The unit is operative and in normal mode.

$F W_{r} / F W_{R}$ : Failed unit waiting for repair/ continuously from previous state.

$S G$ : The server is good.

$F U_{r} / F U_{R}$ : Failed unit under repair/ continuously from previous state.

$S F U_{t} / S F U_{T}$ : Failed server waiting for treatment/ continuously from previous state.

$S F W_{t} / S F W_{T}$ : Failed server under treatment/ continuously from previous state.

$z(t) / Z(t) \lambda \quad:$ pdf/ cdf of failure time of the unit.

$u(t) / U(t) \gamma:$ pdf / cdf of failure time of the server.

$g(t) / G(t) \alpha:$ pdf / cdf of repair time of the failed unit.

$h(t) / H(t) \beta:$ pdf / cdf of the treatment time of the server.

$k(t) / K(t):$ pdf / cdf of the waiting time of the server for treatment.

$q_{i, j}(t) / Q_{i, j}(t):$ pdf / cdf of direct transition time from a regenerative state $i$ to a regenerative state $j$ without visiting any other regenerative state.

$q_{i, j . k}(t) / Q_{i, j . k}(t):$ pdf / cdf of first passage time from a regenerative state $i$ to a regenerative state $j$ or to a failed state $\mathrm{j}$ visiting state $\mathrm{k}$ once in $(0, \mathrm{t}]$.

$q_{i, j . k, r}(t) / Q_{i, j . k, r}(t) \quad$ : pdf / cdf of first passage time from regenerative state $\mathrm{i}$ to a regenerative state $\mathrm{j}$ or to a failed state $\mathrm{j}$ visiting state $\mathrm{k}, \mathrm{r}$ once in $(0, \mathrm{t}]$.

$q_{i, j . k, r, s}(t) / Q_{i, j . k, r, s}(t)$ : pdf / cdf of first passage time from regenerative state $\mathrm{i}$ to a regenerative state $\mathrm{j}$ or to a failed state $\mathrm{j}$ visiting state $\mathrm{k}, \mathrm{r}$ and $\mathrm{s}$ once in $(0, \mathrm{t}]$.

$M_{i}(t)$ : Probability that the system is up initially in state $\mathrm{S}_{\mathrm{i}}$

$\in \mathrm{E}$ is up at time $\mathrm{t}$ without visiting to any other regenerative state.

$W_{i}(t)$ : Probability that the server is busy in the state $S_{\mathrm{i}}$ up to time ' $\mathrm{t}$ ' without making any transition to any other regenerative state or returning to the same state via one or more non-regenerative states. 
$m_{i, j}$ : Contribution to mean sojourn time $\left(\mu_{\mathrm{i}}\right)$ in state $\mathrm{S}_{\mathrm{i}}$ when system transit directly to state $\mathbf{j}$.

$(s) /(c)$ : Stieltjes convolution / Laplace convolution.

$\sim /^{*} \quad$ : Laplace Stieltjes Transform (LST) / Laplace

Transform (LT).

The following are the possible states of the system model

The regenerative states $(E)$ :
$S_{0}=(O, C S), S_{1}=\left(F U_{r}, O, S G\right), S_{2}=\left(F W_{r}, O, S F W_{t}\right)$

Non-regenerative states:

$S_{3}=\left(F W_{R}, O, S F U_{t}\right), S_{4}=\left(F W_{R}, F W_{r}, S F W_{T}\right)$,

$S_{5}=\left(F U_{R}, F W_{r}, S G\right), S_{6}=\left(F W_{R}, F W_{r}, S F U_{T}\right)$,

$S_{7}=\left(F W_{r}, F W_{R}, S F W_{t}\right), S_{8}=\left(F W_{R}, F W_{R}, S F U_{t}\right)$,

$S_{9}=\left(F U_{r}, F W_{R}, S G\right)$

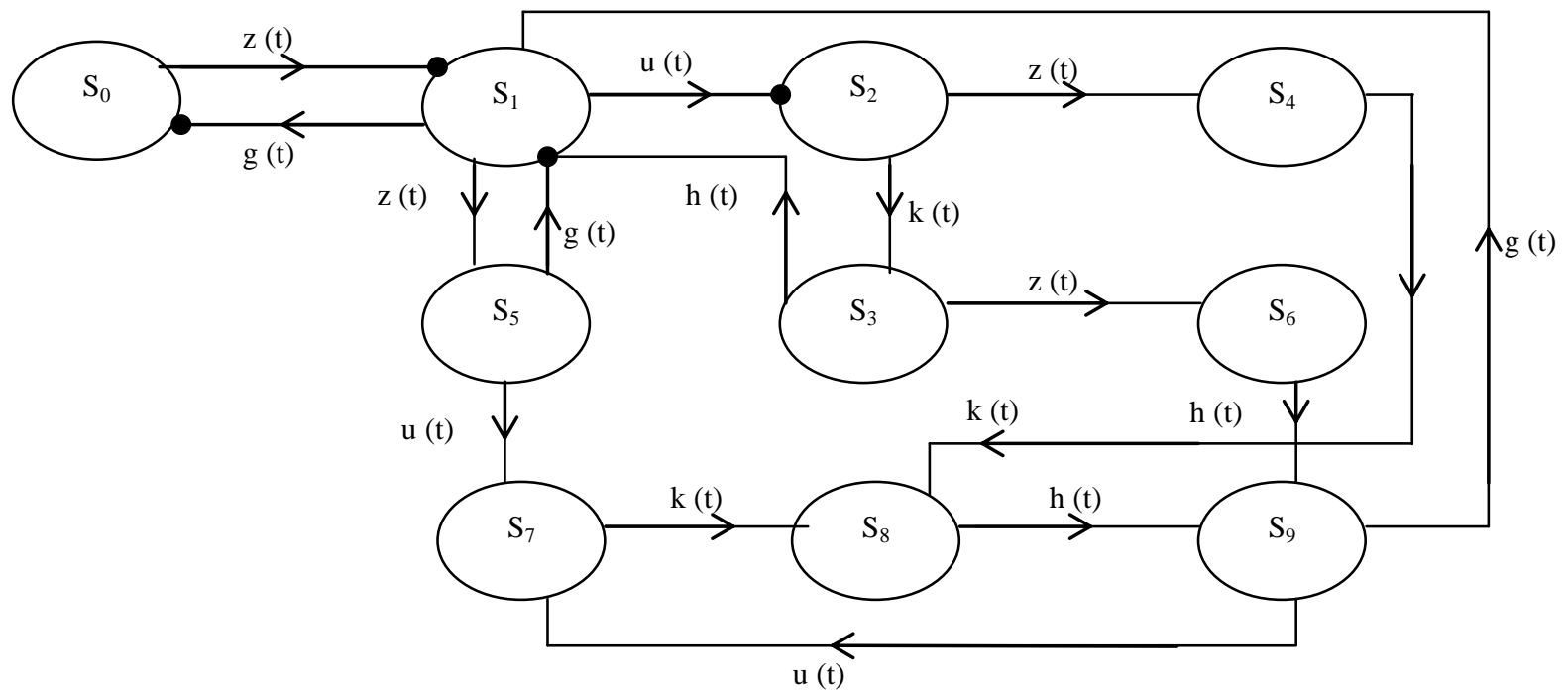

Fig. 1: System State Transition Diagram

\section{THE MODEL ANALYSIS}

\subsection{Transition Probabilities}

Simple probabilistic considerations [20-21], yields the following expressions for the non- zero elements

$p_{i j}=Q_{i j}(\infty)=\int_{0}^{\infty} q_{i j}(t) d t$

as we get

$p_{0,1}=\int_{0}^{\infty} z(t) d t, p_{1,0}=\int_{0}^{\infty} g(t) \bar{Z}(t) \bar{U}(t) d t$,

$p_{1,2}=\int_{0}^{\infty} u(t) \bar{Z}(t) \bar{G}(t) d t, p_{1,5}=\int_{0}^{\infty} z(t) \bar{G}(t) \bar{U}(t) d t$,

$p_{2,4}=\int_{0}^{\infty} z(t) \bar{K}(t) d t, p_{1,1.5}=p_{1,5}(c) p_{5,1}$,

$p_{1,1.5(7,8,9)^{\mathrm{n}}}=p_{1,5}(c) p_{5,7}(c) p_{7,8}(c) p_{8,9}(c) p_{9,1}$,

$p_{2,3}=\int_{0}^{\infty} k(t) \bar{Z}(t) d t, p_{2,1.3}=p_{2,3}(c) p_{3,1}$,

$p_{2,1.3,6,(9,7,8)^{n}}=p_{2,3}(c) p_{3,6}(c) p_{6,9}(c) p_{9,1}$,

$p_{2,1.4,(8,9,7)^{n}}=p_{2,4}(c) p_{4,8}(c) p_{8,9}(c) p_{9,1}$,

$p_{2,6.3}=p_{2,3}(c) p_{3,6}, p_{3,1}=\int_{0}^{\infty} h(t) \bar{Z}(t) d t, p_{3,6}=\int_{0}^{\infty} z(t) \bar{H}(t) d t$,

$p_{4,8}=\int_{0}^{\infty} k(t) d t, p_{5,1}=\int_{0}^{\infty} g(t) \bar{U}(t) d t$,

$p_{5,7}=\int_{0}^{\infty} u(t) \bar{G}(t) d t, p_{6,9}=\int_{0}^{\infty} h(t) d t, p_{7,8}=\int_{0}^{\infty} k(t) d t, p_{8,9}=\int_{0}^{\infty} h(t) d t$,

$p_{9,1}=\int_{0}^{\infty} g(t) \bar{U}(t) d t, p_{9,7}=\int_{0}^{\infty} u(t) \bar{G}(t) d t$

For these Transition Probabilities, it can be verified that

$$
\begin{aligned}
& p_{0,1}=p_{1,0}+p_{1,2}+p_{1,5}=p_{1,0}+p_{1,2}+p_{1,1.5}+ \\
& p_{1,1.5,(7,8,9)^{n}}=p_{2,3}+p_{2,4}=p_{2,1.3}+p_{2,1.3,6,(9,7,8)^{n}}+ \\
& p_{2,1.4,(8,9,7)^{n}}=p_{3,1}+p_{3,6}=p_{4,8}=p_{5,1}+p_{5,7}=p_{6,9}= \\
& p_{7,8}=p_{8,9}=p_{9,1}+p_{9,7}=1
\end{aligned}
$$

\subsection{Mean Sojourn Times}

The Mean sojourn time $\mu_{\mathrm{i}}$ in state $\mathrm{S}_{\mathrm{i}}$ are given by:

$\mu_{i}=E(t)=\int_{0}^{\infty} P(T>t) d t$

$\mu_{0}=\int_{0}^{\infty} \bar{Z}(t) d t, \mu_{1}=\int_{0}^{\infty} \bar{Z}(t) \bar{U}(t) \bar{G}(t) d t, \mu_{2}=\int_{0}^{\infty} \bar{K}(t) \bar{Z}(t) d t$

The unconditional mean time taken by the system to transit from any state $S_{i}$ when time is counted from epoch at entrance into state $S_{\mathrm{j}}$ is stated as:

$$
\begin{aligned}
& m_{i j}=\int t d Q_{i j}(t)=-q_{i j}^{* \prime}(0) \\
& m_{0,1}=\mu_{0}, m_{1,0}+m_{1,2}+m_{1,5}=\mu_{1}, m_{1,0}+m_{1,2}+ \\
& m_{1,1.5}+m_{1,1.5,(7,8,9)^{n}}=\mu_{1}^{\prime}, m_{2,3}+m_{2,4}=\mu_{2}, \\
& m_{2,1.3}+m_{2,1.3,6,(9,7,8)^{n}}+m_{2,1.4,(8,9,7)^{n}}=\mu_{2}^{\prime}, \\
& m_{3,1}+m_{3,6}=\mu_{3}, m_{4,8}=\mu_{4}, m_{5,1}+m_{5,7}=\mu_{5}, \\
& m_{6,9}=\mu_{6}, m_{7,8}=\mu_{7}, m_{8,9}=\mu_{8}, m_{9,1}+m_{9,7}=\mu_{9}
\end{aligned}
$$

\subsection{Mean Time To System Failure}

Let $\phi_{i}(t)$ be the c.d.f of the first passage time from regenerative state $S_{i}$ to a failed state. Regarding the failed state as absorbing state, the following recursive relations are obtained for $\phi_{i}(t)$ : 


$$
\begin{aligned}
\phi_{i}(t)= & \left.\sum_{j} Q_{i, j}(t)+Q_{i, j . k}(t)+Q_{i, j . k l}(t)+\ldots \ldots \ldots+Q_{i, j . k l m \ldots \ldots . . .}(t)\right\} \\
& (c) \phi_{j}(t)+\sum_{f} Q_{i, f}(t) ; i=0,1,2
\end{aligned}
$$

Taking LST of equation (3) and solving for $\tilde{\phi}_{0}(s)$, the MTSF is given as follows:

$$
\begin{aligned}
& \operatorname{MTSF}=\lim _{s \rightarrow 0} R^{*}(s)=\lim _{s \rightarrow 0}\left[\frac{\left\{1-\tilde{\phi}_{0}(s)\right\}}{s}\right] \\
& =\frac{\mu_{0}\left[1-p_{1,2} p_{2,1.3}\right]+\mu_{1}+\mu_{2}^{\prime \prime} p_{1,2}}{1-p_{0,1} p_{1,0}-p_{1,2} p_{2,1.3}}
\end{aligned}
$$

The reliability $\mathrm{R}(\mathrm{t})$ is given by

$$
R(t)=L^{-1}\left\{R^{*}(s)\right\}=L^{-1}\left[\frac{\left\{1-\tilde{\phi_{0}}(s)\right\}}{s}\right]
$$

\section{COST-BENEFIT ANALYSIS}

\subsection{Steady State Availability}

Let $A_{i}(t)$ be the probability that the system is in up-state at an instant ' $t$ ' given that the system entered regenerative state $\mathrm{S}_{\mathrm{i}}$ at $\mathrm{t}=0$. The recursive relations for $A_{i}(t)$ are as follows:

$$
\begin{aligned}
A_{i}(t)= & M_{i}(t)+\sum_{j}\left\{q_{i, j}(t)+\delta_{i, j . k l \ldots . .}\left\{q_{i, j . k}(t)\right.\right. \\
& \left.\left.+q_{i, j . k l}(t)+\ldots \ldots . . .\right\}\right\}(c) A_{j}(t) ; i=0,1,2
\end{aligned}
$$

Let us define

$\delta_{i, j . k l \ldots}=\left\{\begin{array}{rr}1 ; & \text { if thereis transition from statei to } \mathrm{j} \text { via } \mathrm{k}, 1 \ldots . \\ 0 & ;\end{array}\right.$ $M_{i}(t)$ is the probability that the system is up initially in state $S_{i} \in E$ is up at time $\mathrm{t}$ without visiting to any other regenerative state, so that

$M_{0}=\int_{0}^{\infty} \bar{Z}(t) d t, M_{1}, M_{1}=\int_{0}^{\infty} \bar{Z}(t) \bar{U}(t) \bar{G}(t) d t$,

$M_{2}=\int_{0}^{\infty} \bar{K}(t) \bar{Z}(t) d t$

Taking LT of equation (4) and solving for $A_{0}^{*}(s)$, the steady state availability is given by

$$
A_{0}(\infty)=\lim _{s \rightarrow 0} s A_{0}^{*}(s)=\frac{\mu_{0} p_{1,0}+\mu_{1}+\mu_{2} p_{1,2}}{\mu_{0} p_{1,0}+\mu_{1}^{\prime}+\mu_{2}^{\prime} p_{1,2}}
$$

\subsection{The Server's Busy Period}

Let $B_{i}(t)$ be the probability that the server is busy in repair of the unit at an instant $\mathrm{t}$ given that the system entered regenerative state $\mathrm{S}_{\mathrm{i}}$ at $\mathrm{t}=0$. The recursive relations for $B_{i}(t)$ are as follows:

$$
\begin{aligned}
B_{i}(t) & =W_{i}(t)+\sum_{i}\left\{q_{i, j}(t)+\delta_{i, j, k, l \ldots \ldots \ldots}\left\{q_{i, j . k}(t)\right.\right. \\
& \left.\left.+q_{i, j . k l}(t)+\ldots \ldots . .\right\}\right\}(c) B_{j}(t) ; i=0,1,2
\end{aligned}
$$

$W_{i}(t)$ be the probability that the server is busy in state $\mathrm{S}_{\mathrm{i}}$ due to repair of the unit up to time ' $t$ ' without making any transition to any other regenerative state or returning to the same via one or more non-regenerative state and so

$$
\begin{aligned}
W_{2}= & \bar{Z}(t) \bar{U}(t) \bar{G}(t)+(z(t) \bar{U}(t) \bar{G}(t)(c) 1) \bar{G}(t)+ \\
& (z(t) \bar{U}(t) \bar{G}(t)(c) u(t) \bar{G}(t)(c) 1) \bar{K}(t)+ \\
& (z(t) \bar{U}(t) \bar{G}(t)(c) u(t) \bar{G}(t)(c) k(t)(c) 1) \bar{H}(t) \\
& +(z(t) \bar{U}(t) \bar{G}(t)(c) u(t) \bar{G}(t)(c) k(t)(c) h(t)(c) 1) \bar{G}(t)
\end{aligned}
$$

Taking LT, of equation (5) and solving for $B_{0}^{*}(s)$, the time for which server is busy due to repair of unit is given by

$$
B_{0}=\lim _{s \rightarrow 0} s B_{0}^{*}(s)=\frac{W_{1}^{*}(0)}{\mu_{0} p_{1,0}+\mu_{1}^{\prime}+\mu_{2}^{\prime} p_{1,2}}
$$

\subsection{Expected Number Of Repairs}

Let $D_{i}(t)$ be the expected number of repairs of the unit in $(0, t]$ given that the system entered regenerative state $S_{i}$ at $t=0$. The recursive relations for $D_{i}(t)$ are as follow:

$$
\begin{aligned}
D_{i}(t)= & \sum_{j}\left\{Q_{i, j}(t)+\delta_{i, j . k l \ldots \ldots . . .}\left\{Q_{i, j . k}(t)\right.\right. \\
& \left.\left.+Q_{i, j . k l}(t)+\ldots \ldots . .\right\}\right\}(s)\left\{\delta_{j}+D_{j}(t)\right\} ; i=0,1,2(6)
\end{aligned}
$$

Using LT, of equation (6) and solving for $\tilde{D}_{0}(s)$, the expected number of repair of the unit are given by

$$
D_{0}=\lim _{s \rightarrow 0} s \tilde{D}_{0}(s)=\frac{1-p_{1,2} p_{2,1.3}}{\mu_{0} p_{1,0}+\mu_{1}^{\prime}+\mu_{2}^{\prime} p_{1,2}}
$$

\subsection{Expected Number Of Treatments}

Let $T_{i}(t)$ be the expected number of treatments given to the server in $(0, \mathrm{t}]$ given that the system entered regenerative state $\mathrm{S}_{\mathrm{i}}$ at $\mathrm{t}=0$. The recursive relations for $T_{i}(t)$ are as follow:

$$
\begin{aligned}
T_{i}(t)= & \sum_{j}\left\{Q_{i, j}(t)+\delta_{i, j . k l \ldots . . .}\left\{Q_{i, j . k}(t)\right.\right. \\
& \left.\left.+Q_{i, j . k l}(t)+\ldots \ldots . .\right\}\right\}(s)\left\{\delta_{j}+T_{j}(t)\right\} ; i=0,1,2
\end{aligned}
$$

Using LT, of equation (7) and solving for $\tilde{T}_{0}(s)$, the expected number of the treatments given to the server are given by

$$
T_{0}=\lim _{s \rightarrow 0} s \tilde{T_{0}}(s)=\frac{p_{2,1.3} p_{1,2}}{\mu_{0} p_{1,0}+\mu_{1}^{\prime}+\mu_{2}^{\prime} p_{1,2}}
$$

\section{COST ANALYSIS}

Let $X_{i}(t)$ denote the measure of $i^{\text {th }}$ characteristic of the system in $(0, \mathrm{t}]$ and $C_{i}$ be its coefficient then the profit incurred to the system model in $(0, \mathrm{t}]$ is given by

$$
P(t)=K_{0} A_{0}(t)-\sum_{i=1}^{3} C_{i} X_{i}(t)
$$

Asymptotically $\left(\right.$ i.e. $\left.t_{\rightarrow \infty}\right)$, we obtain

$$
\begin{aligned}
& \operatorname{Profit}, P_{0}=\underset{t \rightarrow \infty}{\operatorname{Lim}} P(t)=\operatorname{Lim}_{t \rightarrow \infty}\left[K_{0} A_{0}(t)-\sum_{i=1}^{3} C_{i}\left\{X_{i}(t)\right\}\right] \\
& =K_{0} A_{0}-\sum_{i=1}^{3} C_{i}\left\{X_{i}(\infty)\right\}
\end{aligned}
$$

$X_{i}(\infty)=\left\{\begin{array}{l}B_{0} ; \text { for } i=1 \\ D_{0} ; \text { for } i=2 \\ T_{0} ; \text { for } i=3\end{array}\right.$

And

$K_{0}=$ Revenueper unituptime of thesystem 
$C_{1}=$ Cost per unit timefor which serveris busy.

$C_{2}=$ Cost per unit timefor the repairof the uint

$C_{3}=$ Cost per unit timefor the server treatment.

and $A_{0}, B_{0}, D_{0}, T_{0}$ are alreadydefined.

\section{SIMULATION STUDY}

Without loss of generality, just for the sake of convenience, let us suppose all the random variables follow exponential distribution i.e.

$z(t)=\lambda e^{-\lambda t}, g(t)=\alpha e^{-\alpha t}, u(t)=\gamma e^{-\lambda t}$,

$k \xi(t)=\xi e^{-\xi t}, h(t)=\beta e^{-\beta t}$

Substituting these values in equations [1-7], the following results are obtained:

\section{Transition Probabilities}

$$
\begin{aligned}
& p_{0,1}=1, p_{1,0}=\frac{\alpha}{(\lambda+\gamma+\alpha)}, p_{1,2}=\frac{\gamma}{(\lambda+\gamma+\alpha)}, \\
& p_{1,5}=\frac{\lambda}{(\lambda+\gamma+\alpha)}, p_{1,1.5}=\frac{\lambda \alpha}{(\lambda+\gamma+\alpha)(\gamma+\alpha)} \\
& p_{1,1.5,(7,8,9)^{n}}=\frac{\lambda \gamma}{(\lambda+\gamma+\alpha)(\gamma+\alpha)}, p_{2,3}=\frac{\xi}{(\lambda+\xi)}, \\
& p_{2,4}=\frac{\lambda}{(\lambda+\xi)}, p_{2,1.3}=\frac{\xi \beta}{(\lambda+\xi)(\beta+\lambda)}, \\
& p_{2,1.3,6,(9,7,8)^{n}}=\frac{\xi \lambda}{(\lambda+\xi)(\beta+\lambda)}, p_{2,1.4,(8,9,7)^{n}}=\frac{\lambda}{(\lambda+\xi)}, \\
& p_{2,6.3}=\frac{\xi \lambda}{(\lambda+\xi)(\beta+\lambda)}, p_{3,1}=\frac{\beta}{(\lambda+\beta)}, \\
& p_{3,6}=\frac{\lambda}{(\lambda+\beta)}, p_{4,8}=1, p_{5,1}=\frac{\alpha}{(\alpha+\gamma)}, \\
& p_{5,7}=\frac{\gamma}{(\alpha+\gamma)}, p_{6,9}=1, p_{7,8}=1, p_{8,9}=1, \\
& p_{9,1}=\frac{\alpha}{(\alpha+\gamma)}, p_{9,7}=\frac{\gamma}{(\alpha+\gamma)}
\end{aligned}
$$

Mean Sojourn Times $\left(\mu_{i}\right)$ for the state $s_{i}$ are

$\mu_{0}=\frac{1}{\lambda}, \mu_{1}=\frac{1}{(\lambda+\alpha+\gamma)}, \mu_{2}=\frac{1}{(\xi+\lambda)}$

MTSF $=\frac{N_{1}}{D_{1}}$, Availability $=\frac{N_{2}}{D_{2}}$

Busy Period of the server $=\frac{N_{3}}{D_{2}}$

Expected Number of Repairs $=\frac{N_{4}}{D_{2}}$,
Expected number of server Treatments $=\frac{N_{5}}{D_{2}}$,

Where

$$
\begin{aligned}
& (\lambda+\xi)^{2}(\lambda+\beta)[2 \lambda+\alpha+\gamma]-(\lambda+\xi) \gamma \xi \beta+ \\
N_{1}= & \frac{\lambda \gamma[\xi(2 \lambda+\beta+\xi)+\lambda(\lambda+\beta)]}{(\lambda+\alpha+\gamma)(\lambda+\xi)^{2}(\lambda+\beta) \lambda} \\
D_{1}= & \frac{(\lambda+\xi)(\lambda+\beta)(\lambda+\gamma)-\gamma \xi \beta}{(\lambda+\alpha+\gamma)(\lambda+\xi)(\lambda+\beta)}, \\
N_{2}= & \frac{(\lambda+\xi)(\lambda+\alpha)+\lambda \gamma}{\lambda(\lambda+\xi)(\lambda+\alpha+\gamma)} \\
& (\lambda+\beta)^{2}(\lambda+\xi)^{2} \xi \beta \alpha(\lambda+\alpha+\gamma)[\alpha(\alpha+\gamma)+ \\
& \lambda(\lambda+\alpha+\gamma)]+\lambda(\lambda+\beta)^{2}(\lambda+\xi)^{2} \lambda \gamma(\lambda+\alpha+\gamma) \\
& {[(\alpha+\gamma)(\beta+\xi)+\xi \beta]+\lambda(\lambda+\alpha+\gamma) \gamma(\alpha+\gamma) } \\
& \{\lambda(\lambda+\xi)(\lambda+\beta)[\xi \beta(\xi-\alpha)+(\alpha+\gamma)(\beta+\xi) \\
& (\xi+(\lambda+\beta))]+(\lambda+\beta) \xi \beta[\lambda(\lambda+\beta)(\lambda+\alpha+\xi) \\
D_{2}= & \frac{+\xi \alpha(2 \lambda+\beta+\xi)]\}}{\lambda(\lambda+\alpha+\gamma)^{2}(\alpha+\gamma)(\lambda+\beta)^{2}(\lambda+\xi)^{2} \alpha \beta \xi} \\
N_{4}= & \frac{(\lambda+\alpha+\gamma)(\lambda+\xi)(\lambda+\beta)-\gamma \xi \beta}{(\lambda+\alpha+\gamma)(\lambda+\xi)(\lambda+\beta)}, \\
N_{5}= & \frac{\gamma \xi \beta}{(\lambda+\alpha+\gamma)(\lambda+\xi)(\lambda+\beta)} \\
N_{3}= & \frac{\left.(\alpha+\gamma+\xi) \xi \xi^{2}\right\}}{(\lambda+\alpha+\gamma)(\lambda+2 \alpha+\gamma)(\lambda+\alpha+\gamma+\xi)(\alpha+\gamma+\xi)} \\
& (\lambda+\alpha+\gamma+\beta)(\alpha+\gamma+\beta)(\xi+\beta)(2 \alpha+\gamma) \\
& (\xi+\alpha)(\beta+\alpha) \alpha \xi \beta \\
& (\alpha \beta(\lambda+\alpha+\gamma+\xi)(\alpha+\gamma+\xi)(\lambda+\alpha+\gamma+\beta) \\
& {[(\lambda+2 \alpha+\gamma) \alpha+(\lambda+\alpha+\gamma) \lambda]+(\lambda+\alpha+\gamma) \lambda \xi \beta\} } \\
+ & (\lambda+\alpha+\gamma)(\lambda+2 \alpha+\gamma)(2 \alpha+\gamma)(\xi+\alpha)(\beta+\alpha) \lambda \gamma \alpha \\
& (\lambda+\alpha+\gamma+\beta)(\alpha+\gamma+\beta)(\xi+\beta) \beta+(\lambda+\alpha+\gamma+\xi) \\
& (\lambda+\beta)\{(2 \alpha+\gamma)(\xi+\alpha)(\beta+\alpha) \\
&
\end{aligned}
$$

The results are shown in the table $1 \& 2$ for numerical simulation assigning the following set of presumed values to various parameters:

$$
\begin{aligned}
& \lambda=0.008, \alpha=0.3, \\
& \gamma=0.02, \xi=0.08, \\
& \mathrm{~K}_{0}=2000 Q \mathrm{C}_{1}=500, \\
& C_{2}=300, \mathrm{C}_{3}=900
\end{aligned}
$$

Tables 1 effect of $\alpha$ and $\psi$ on system performance w.r.t server treatment rate $\beta(\lambda=0.008, \gamma=0.02)$

\begin{tabular}{lllll}
\hline $\begin{array}{l}\text { Performance } \\
\text { Index }\end{array}$ & $\beta$ & $\begin{array}{l}\alpha=0.3 \\
\xi=0.08\end{array}$ & $\begin{array}{l}\alpha=0.4 \\
\xi=0.08\end{array}$ & $\begin{array}{l}\alpha=0.3 \\
\xi=0.09\end{array}$ \\
\hline & & 2345.09 & 3043.454 & 2357.225 \\
MTSF & 0.01 & 2747.837 & 3580.45 & 2770.082 \\
& 0.02 & 2998.072 & 3914.095 & 3027.888 \\
& 0.03 & 3168.632 & 4141.509 & 3204.18 \\
& 0.04 & 3292.345 & 4306.46 & 3332.34 \\
& 0.05 & & & \\
& & & & \\
& & &
\end{tabular}




\begin{tabular}{|c|c|c|c|c|}
\hline \multirow{5}{*}{ Availability } & 0.01 & 0.945862 & 0.959394 & 0.946001 \\
\hline & 0.02 & 0.971519 & 0.978844 & 0.971672 \\
\hline & 0.03 & 0.980342 & 0.98548 & 0.980498 \\
\hline & 0.04 & 0.984802 & 0.988825 & 0.98496 \\
\hline & 0.05 & 0.987492 & 0.99084 & 0.987651 \\
\hline \multirow{5}{*}{ Profit } & 0.01 & 18901.74 & 19175.57 & 18904.51 \\
\hline & 0.02 & 19414.73 & 19564.46 & 19417.78 \\
\hline & 0.03 & 19591.11 & 19697.14 & 19594.24 \\
\hline & 0.04 & 19680.27 & 19764.01 & 19683.42 \\
\hline & 0.05 & 19734.06 & 19804.3 & 19737.23 \\
\hline
\end{tabular}

Table 2 effect of $\lambda$ and $\gamma$ on system performance w.r.t server treatment rate $\beta(\alpha=0.3, \xi=0.08)$

\begin{tabular}{|c|c|c|c|c|}
\hline \multirow{2}{*}{$\begin{array}{l}\text { Performance } \\
\text { Index }\end{array}$} & \multirow[t]{2}{*}{$\beta$} & $\lambda=0.008$ & $\lambda=0.009$ & $\lambda=0.008$ \\
\hline & & $\gamma=0.02$ & $\gamma=0.02$ & $\gamma=0.04$ \\
\hline \multirow{5}{*}{ MTSF } & 0.01 & 2345.09 & 1928.287 & 1599.019 \\
\hline & 0.02 & 2747.837 & 2230.048 & 1952.535 \\
\hline & 0.03 & 2998.072 & 2419.378 & 2193.824 \\
\hline & 0.04 & 3168.632 & 2549.243 & 2369.007 \\
\hline & 0.05 & 3292.345 & 2643.857 & 2501.977 \\
\hline \multirow{5}{*}{ Availability } & 0.01 & 0.945862 & 0.939205 & 0.895309 \\
\hline & 0.02 & 0.971519 & 0.9678 & 0.944193 \\
\hline & 0.03 & 0.980342 & 0.977682 & 0.961472 \\
\hline & 0.04 & 0.984802 & 0.982687 & 0.970286 \\
\hline & 0.05 & 0.987492 & 0.98571 & 0.975628 \\
\hline \multirow{5}{*}{ Profit } & 0.01 & 18901.74 & 18766.76 & 17890.84 \\
\hline & 0.02 & 19414.73 & 19338.46 & 18868.16 \\
\hline & 0.03 & 19591.11 & 19536.02 & 19213.58 \\
\hline & 0.04 & 19680.27 & 19636.07 & 19389.79 \\
\hline & 0.05 & 19734.06 & 19696.5 & 19496.56 \\
\hline
\end{tabular}

\section{EXAMPLE}

For illustration a cold standby system is considered with values for different parameters as taken previously in the simulation study with $\beta=0.09$, and following results are obtained for different measures of system performance:

\section{MTSF $=3567.876$}

\section{Availability $=0.9911$}

Busy period due to repair $=0.026124$

Expected number of repairs $=0.007976$

Expected number of treatments of the server $=0.000428$

System profit $=19830.21$

\section{DISCUSSION ON RESULTS}

Table1 shows the effect of $\alpha$ and $\xi$ on different measures of system performance, for fixed values of $\lambda$ and $\gamma$. It indicates how MTSF, availability and profit rise with increasing $\beta$. It reveals that the system performance improves considerably with increasing treatment rate of the server. For constant values of all other parameters, as $\alpha$ increases from 0.3 to 0.4 all the system performance measures exhibit uprising trends. It means the higher the repair rate of unit better is the system performance. The similar trend occurs for increasing $\xi$ from 0.08 to 0.09 , though slower than that of $\alpha$. The effect of $\lambda$ and $\gamma$ on system performance w.r.t server treatment rate $\beta$ for fixed levels of $\alpha \& \xi$, is shown in table 2 . The system performance declines with increasing values of both of these parameters, for all constant set of values of other parameters. This trend shows that the failure of both unit as well as that of server undesirably affect system performance. Further, the effect of $\gamma$ is worse even than that of $\lambda$. As the server looks after the failed unit thus if it fails then both the mean time to system failure as well as availability and hence the profit declines.

\section{CONCLUDING REMARKS}

This paper develops a probabilistic model of a cold standby system with the possibility of server failure getting delayed treatment thereafter. The operative unit, cold standby unit, state of server, failure of unit, failure of server, repair of unit and treatment of server are the key concerns of the model. It uses the semi-Markov approach of stochastic processes to build the model and identifies different renewal-points for deriving and analyzing the expressions for transition probabilities and system performance measures. The simulation results obtained for a particular case highlight the importance of study. The results may facilitate the reliability practitioners to understand and improve better system designs.

\section{ACKNOWLEDGEMENTS}

1. The authors are very grateful to anonymous referees for in-depth review and valuable suggestions.

2. This work is a part of major research project [F.N. 42-34/ 2013 (SR)], supported by University Grant Commission (UGC) under Ministry of Human Resource Development, Government of India. 


\section{REFERENCES}

[1] Nakagawa T, Osaki S. 1975. Stochastic behavior of a two-unit priority standby redundant system with repair. Microelectron Reliab. 14(3), 309-13.

[2] Bieth, B., Hong, L., and Sarkar, J. A. 2010. Standby system with two repair persons under arbitrary life-and repair times. Mathematical and Computer Modelling 51(5), 756-767.

[3] Mahmoud, M.A.W. and Moshref, M.E. 2010. On a twounit cold standby system considering hardware, human error failures and preventive maintenance, Mathematical and Computer Modelling 51 (5-6), 736-745.

[4] Bhardwaj, R. K., and Malik, S. C. 2011. Asymptotic performance analysis of 2003 cold standby system with constrained repair and arbitrary distributed inspection time. International Journal of Applied Engineering Research 6 (08), 1493-1502.

[5] Bhardwaj R. K. and Kaur, Komaldeep. 2014. Reliability and Profit Analysis of a Redundant System with Possible Renewal of Standby Subject to Inspection, International Journal of Statistics and Reliability Engineering, 1 (1), $36-46$.

[6] Avi-Itzhak, B \& Naor, P. 1963. Some queuing problems with the service station subject to breakdown, Operations Research 11 (3), 303-320.

[7] Mitrany, I.L. Avi-Itzhak, B. 1968. A many-server queue with service interruptions, Operations Research 16, 628638.

[8] Neuts, M. and Lucantoni, D. 1979. Markovian queue with $\mathrm{N}$-servers subject to breakdowns and repairs, Management Science 25 (9), 849-861.

[9] Federgruen, A. and Green, L. 1986. Queueing systems with service interruptions, Operations Research 34 (5), 752-768.

[10] Lee, D. 1997. Analysis of a single server queue with semi-Markovian service interruption, Queueing Systems 27 (1-2), 153-178.
[11] Yang, X., \& Alfa, A. S., 2009. A class of multi-server queuing system with server failures, Computers \& Industrial Engineering, 56(1), 33-43.

[12] Dhankar, A.K., Bhardwaj, R.K., Malik, S.C., 2012. Reliability Modeling and Profit Analysis of a System with Different Failure Modes and Replaceable Server Subject To Inspection, Int. J. of Statistics and Analysis 2(3), 245-255.

[13] Malik, S. C., Dhankar, A.K.. 2013. Reliability Modeling and Cost-Analysis of a System with Replacement of the server and Unit subject to Inspection", Journal of Statistics and Management Systems, 16 (2-3), 137-162.

[14] Bhardwaj, R.K., Singh, Ravinder, 2014. Semi Markov Approach for Asymptotic Performance Analysis of a Standby System with Server Failure, International Journal of Computer Applications 98 (3), 9-14.

[15] Bhardwaj, R.K., Singh, Ravinder, 2014. Steady State Behavior of a Cold-Standby System with Server Failure and Arbitrary Repair, Replacement \& Treatment", International Journal of Applied Engineering Research 9 (24), 26563-26578

[16] Bhardwaj, R.K., Singh, Ravinder, 2015. Semi-Markov Model of a Standby System with General Distribution of Arrival and Failure Times of Server. American Journal of Applied Mathematics and Statistics, 3 (3), 105-110.

[17] Bhardwaj, R.K., Singh, Ravinder, 2015. An InspectionRepair-Replacement Model of a Stochastic Standby System with Server Failure", Mathematics in Engineering, Science and Aerospace 6 (2), 191-203.

[18] Ross SM. 1996. Stochastic processes. Wiley.

[19] Smith, W. L. 1955. Regenerative stochastic processes. Proceedings of the Royal Society of London. Series A Mathematical and Physical Sciences 232 (1188), 6-31.

[20] Johnson, R. 2015. Miller and Freund's probability and Statistics for Engineers, PHI.

[21] Ross, S. M. 2010. Introduction to probability models. Academic press. 